\title{
A comparison of two gluteus maximus EMG maximum voluntary isometric contraction positions
}

Bret Contreras, Andrew D Vigotsky, Brad J Schoenfeld, Chris Beardsley, John B Cronin

Background: The purpose of this study was to compare the peak electromyography (EMG) of the most commonly-used positions in the literature, the prone bent-leg (90) hip extension against manual resistance applied to the distal thigh (PRONE), to a novel position, the standing glute squeeze (SQUEEZE).

Methods: Surface EMG electrodes were placed on the upper and lower gluteus maximus of thirteen recreationally active females (age $=28.9$ years; height $=164 \mathrm{~cm}$; body mass $=$ $58.2 \mathrm{~kg}$ ), before three maximum voluntary isometric contraction (MVIC) trials for each position were obtained in a randomized, counterbalanced fashion.

Results: No significant ( $p \leq 0.05$ ) differences were observed between PRONE (upper: 91.94\%; lower: 94.52\%) and SQUEEZE (upper: 92.04\%; lower: $85.12 \%$ ) for both the upper and lower gluteus maximus. Neither the PRONE nor SQUEEZE was more effective between all subjects.

Conclusions: In agreement with other studies, no single testing position is ideal for every participant. Therefore, it is recommended that investigators employ multiple MVIC positions, when possible, to ensure accuracy. Future research should investigate a variety of gluteus maximus MVIC positions in heterogeneous samples. 
1 A comparison of two gluteus maximus EMG maximum voluntary isometric contraction

2

3

4

5

6

7

8

9

10

11

12

13

14

15

$16{ }^{5}$ School of Exercise, Biomedical and Health Science, Edith Cowan University, Perth, Australia positions

Running Head: GMax MVIC

Bret Contreras, MA ${ }^{1}$

Andrew D. Vigotsky ${ }^{2}$

Brad J. Schoenfeld, $\mathrm{PhD}^{3}$

Chris Beardsley ${ }^{4}$

John Cronin, $\mathrm{PhD}{ }^{1,5}$

${ }^{1}$ Auckland University of Technology, Sport Performance Research Institute New Zealand

${ }^{2}$ Kinesiology Program, Arizona State University, Phoenix, AZ

${ }^{3}$ Department of Health Sciences, CUNY Lehman College, Bronx, NY

${ }^{4}$ Strength and Conditioning Research Limited, London, UK

17

18

19

20

21

22

23 
25 Maximum voluntary isometric contractions (MVIC) are often used to normalize

26 electromyography (EMG) signals. It is important to employ an MVIC position that elicits the

27 highest activation in order to increase the validity of EMG studies and decrease incidents of

28 abnormally high normalized mean and peak EMG data. In order for accurate comparisons to be

29 made between studies, it is also important for researchers to standardize MVIC positions, or at

30 least use positions that elicit similar magnitudes of EMG activity. A number of MVIC positions

31 have been used in the literature to assess the gluteus maximus, including the Biering-Sorenson

32 position (Cambridge et al. 2012; McGill et al. 2009), the prone straight leg hip extension position

33 (Barton et al. 2014; Worrell et al. 2001), the prone bent leg position (Jakobsen et al. 2013;

34 Youdas et al. 2013), the prone straight leg position with $70^{\circ}$ of hip flexion (Simenz et al. 2012),

35 and the standing bent leg position (Boudreau et al. 2009). The most commonly used position,

36 however, is the prone bent-leg $\left(90^{\circ}\right)$ hip extension with manual resistance applied to the distal

37 thigh (PRONE) (Choi et al. 2014; Emami et al. 2014; Hislop et al. 2013; Kang et al. 2013;

38 Kendall et al. 1993; Oh et al. 2007).

A recent study by Simenz et al. (2012) that used a prone gluteus maximus MVIC position in $70^{\circ}$

41 of hip flexion, demonstrates the importance of standardizing MVIC positions across studies.

42 Researchers have shown that lower gluteus maximus amplitude is elicited at higher degrees of

43 hip flexion and reaches a maximum EMG amplitude at end range hip extension (Worrell et al.

44 2001). By employing an MVIC position that renders significantly lower EMG activity than those

45 values that are truly maximal, the normalized data of Simenz et al. (2012) are most likely

46 overestimated. For example, if the work of Worrell et al. (2001) is extrapolated, the MVIC 
47 position used by Simenz et al. (2012) would only elicit approximately $80 \%$ of true MVIC,

48 translating into $25 \%$ greater mean and peak values when compared to the true MVIC position.

49 The data reported by Simenz et al. (2012) therefore cannot be used for comparison with exercises

50 in other studies that utilized alternative MVIC positions with smaller hip flexion angles, as the

51 data would have overestimated how effectively the gluteus maximus was activated. Therefore, it

52 is apparent that researchers should only compare EMG data that utilize positions that render

53 similar values.

55 Since Worrell et al. (2001) found that full hip extension elicited the greatest amount of gluteus maximus EMG activity, and this finding is corroborated by earlier work from Wheatley \&

57 Jahnke (1951) and Fischer \& Houtz (1968), it is postulated that the most appropriate gluteus maximus MVIC position is at full hip extension, or hip hyperextension. PRONE is currently the recommended position in several texts on muscle testing (Hislop et al. 2013; Kendall et al.

60 1993), although to the authors' knowledge, this position has not been compared to others in the

61 literature. In order to correct for individual variation, some researchers have employed multiple

62 MVIC positions. For example, McGill et al. (2009) used both the Biering-Sorenson and PRONE 63 positions; whichever position elicited the greatest activity was used for normalization purposes.

64 The authors, however, are unaware of any existing research that quantitatively compares gluteus 65 maximus MVIC positions.

67 The gluteus maximus muscle appears to be segmented into at least two subdivisions, which may 68 display different EMG activity in response to certain muscle actions. McAndrew et al. (2006) 69 used a laser-based mechanomyographic (MMG) technique to measure the mean contraction time 
70 in six subdivisions of the gluteus maximus, both in the sagittal plane (superior, middle, inferior)

71 and in the frontal plane (medial and lateral). The superior region displayed the longest

72 contraction time followed by the middle region and then the inferior region. On the basis of these

73 findings, McAndrew et al. (2006) suggested that the superior region may contain more slow

74 twitch fibers and be more involved in postural tasks compared to the inferior region, while the

75 inferior region may contain more fast twitch fibers and be more involved in dynamic tasks. This

76 is further substantiated by the work of Lyons et al. (1983) and Karlsson \& Jonsson (1965), who

77 found differences between upper and lower gluteus maximus EMG during functional movement;

78 for example, load acceptance during stair ambulation better targets the lower gluteus maximus

79 (Lyons et al. 1983), while hip abduction better targets the upper gluteus maximus (Karlsson \&

80 Jonsson 1965).

81

82 Pilot data from our lab showed that some subjects were able to elicit greater EMG activity during

83 a standing glute squeeze (SQUEEZE) when compared to PRONE, and this was especially true

84 for the upper gluteus maximus. Given this observation and the findings articulated in previous

85 paragraphs, the purpose of this investigation was to compare upper and lower gluteus maximus

86 EMG activity in PRONE versus SQUEEZE. Based on our pilot data, it was hypothesized that

87 SQUEEZE would elicit greater upper gluteus maximus EMG activity, while PRONE would

88 elicit greater lower gluteus maximus EMG activity. 
$91 \quad \underline{\text { Subjects }}$

92 Thirteen healthy women (age $=28.9 \pm 5.1$ years; height $=164 \pm 6.3 \mathrm{~cm}$; body mass $=58.2 \pm 6.4$

$93 \mathrm{~kg}$ ) with $7.0 \pm 5.8$ years of resistance training experience participated in this study. Inclusion

94 criteria required subjects to be between 20 to 40 years of age and have at least 3 years of

95 consistent resistance training experience. All subjects were healthy and free of any

96 musculoskeletal or neuromuscular injuries, pain, or illnesses. Subjects completed an Informed

97 Consent form. Subjects were advised to refrain from training their lower body for 72 hours prior

98 to testing. The study was approved by the Auckland University of Technology Ethics Committee

99 (AUTEC Reference number 13/375).

100

101 Procedures

102 Subjects first performed a 10-minute general warm-up consisting of various dynamic stretches

103 for the lower body musculature. Following warm-up, subjects practiced each testing position

104 several times, until they felt comfortable with the technique. Subjects were asked to wear

105 appropriate clothing for access to the EMG electrode placement sites. Before placing the

106 electrodes on the skin, excess hair was removed with a razor, and skin was cleaned and abraded

107 using an alcohol swab. After preparation, self-adhesive disposable silver/silver chloride pre-

108 gelled dual snap surface bipolar electrodes (Noraxon Product \#272, Noraxon USA Inc,

109 Scottsdale, AZ) with a diameter of 1 centimeter $(\mathrm{cm})$ and an inter-electrode distance of $2 \mathrm{~cm}$

110 were attached in parallel to the fibers of the right upper and lower gluteus maximus. More

111 specifically, "[upper gluteus maximus] electrodes were placed two finger's width above the line

112 just under the spina iliaca posterior superior and the trochanter major; [lower gluteus maximus] 
113 electrodes were set below the same line" (Fujisawa et al. 2014) (Figure 1, Figure 2). After the

114 electrodes were secured, a quality check was performed to ensure EMG signal validity.

115

116 Following electrode placement, subjects completed three trials of PRONE then SQUEEZE, or

117 vice versa. The PRONE position was performed by having the subject lie prone on the bench,

118 flexing their knee to $90^{\circ}$, and was told to extending her hip while manual resistance was applied

119 to the distal thigh (Figure 1). The SQUEEZE position was performed by having the subject stand

120 with her feet slightly wider than shoulder width apart and hips slightly externally rotated. The

121 subject was instructed to squeeze her glutei and focus on externally rotating and extending her

122 hips (Figure 2). For example, if a subject was randomized to complete PRONE first, her testing

123 order would be PRONE, SQUEEZE, rest, PRONE, SQUEEZE, rest, PRONE, SQUEEZE. Each

124 contraction phase lasted 5 seconds, and each rest phase lasted 3 minutes. Randomization was

125 counterbalanced so that half the subjects performed PRONE first and the other half performed

126 SQUEEZE first. In all MVIC positions, subjects were instructed to contract the gluteus maximus

127 "as hard as possible." 


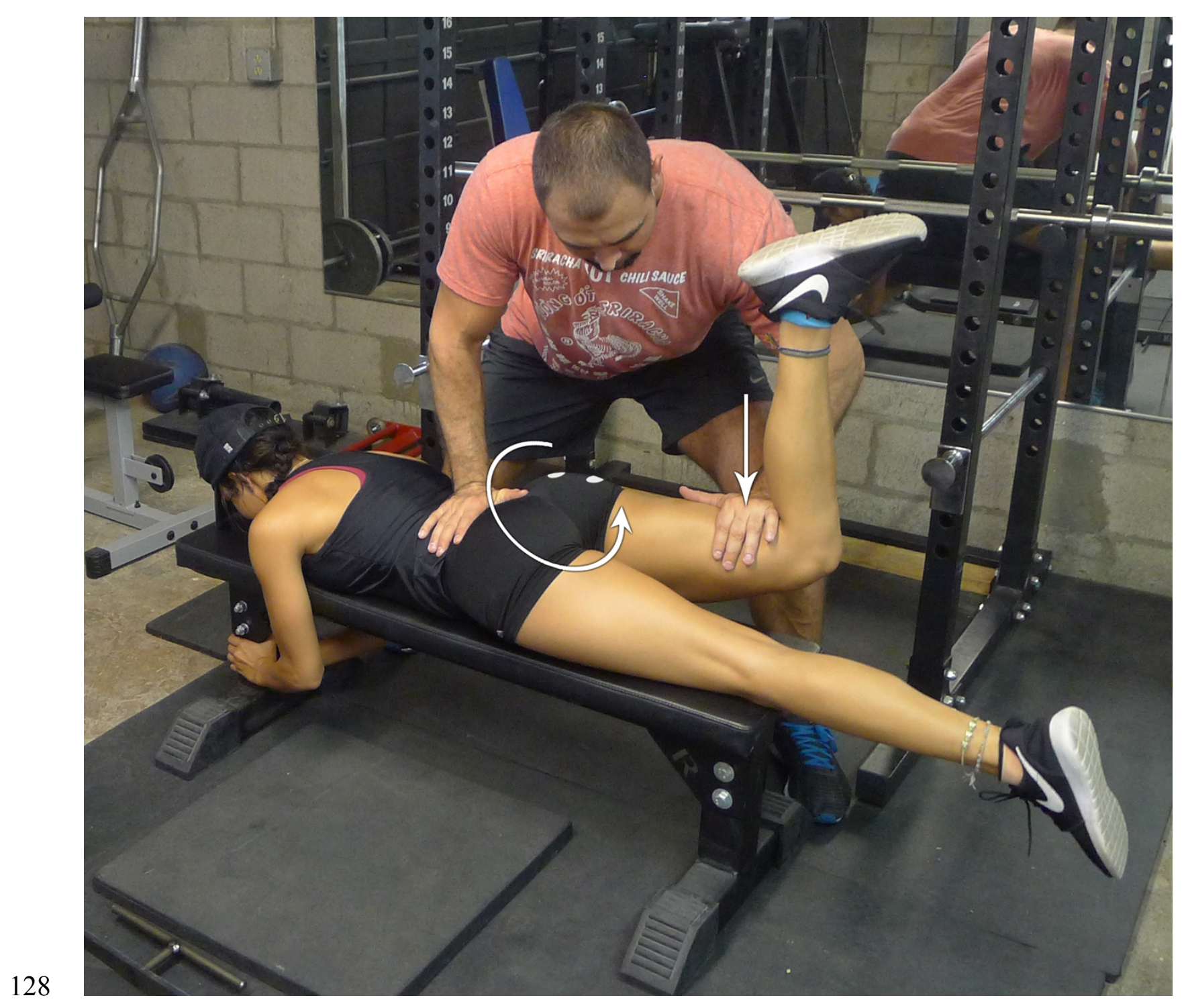

129 Figure 1. Prone bent-leg hip extension position. Force is applied to the distal thigh while the

130 subject produces a hip extension moment. 


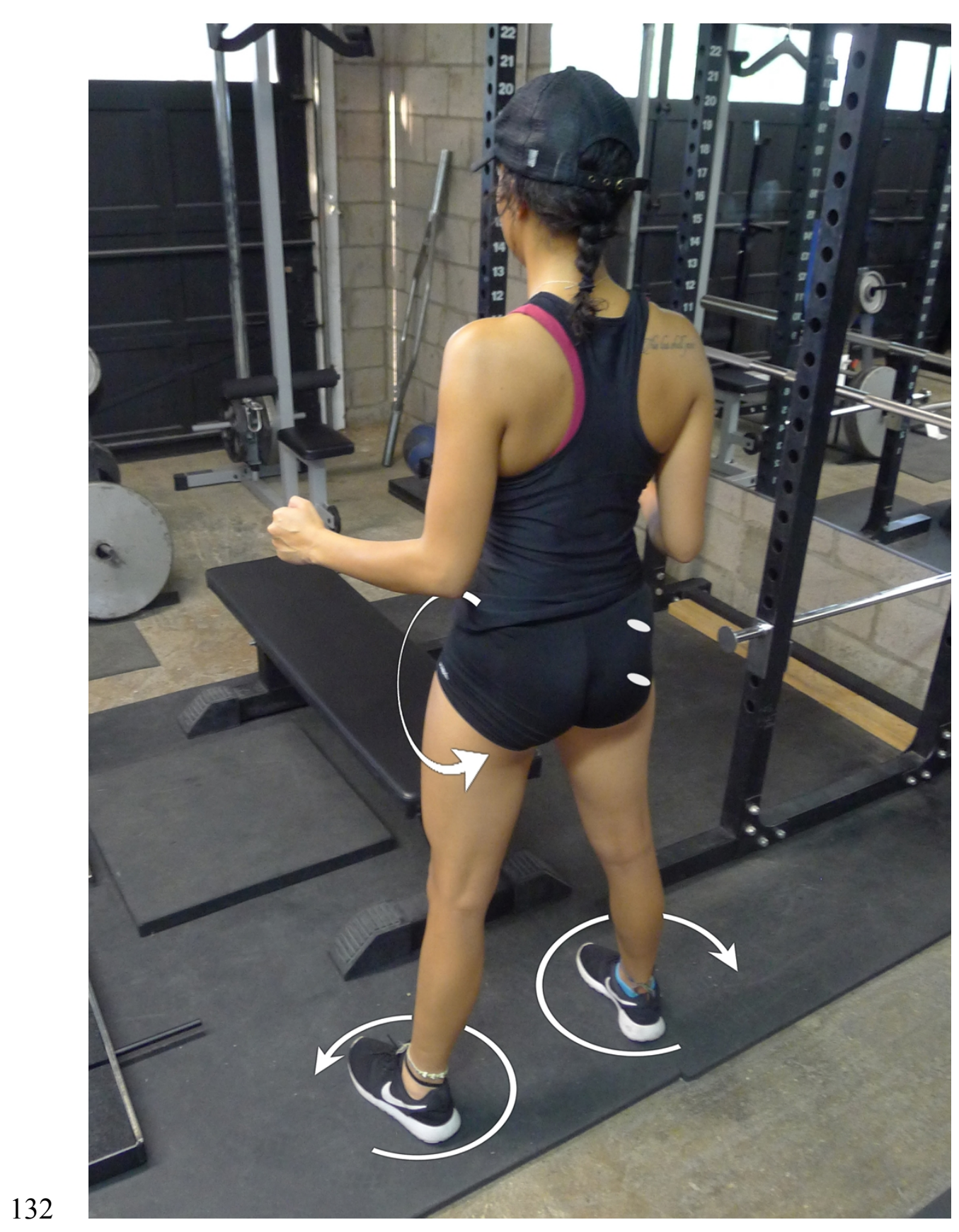

133 Figure 2. Standing glute squeeze position. The subject squeezes her glutei, which generates hip

134 extension and external rotation moments.

136 Raw EMG signals were collected at $2000 \mathrm{~Hz}$ by a Myotrace 400 EMG unit (Noraxon USA Inc,

137 Scottsdale, AZ). Data was sent in real time to a computer via Bluetooth and recorded and 
138 analyzed by MyoResearch 3.6 Clinical Applications software (Noraxon USA, Inc., Scottsdale,

139 AZ). A 10-500 Hz bandpass filter was applied to EMG data. Signals of all MVIC trials were full-

140 wave rectified and smoothed with a root mean square (RMS) algorithm with a 100 ms window.

141 Maximal peak EMG values over a $1000 \mathrm{~ms}$ window, or the $1000 \mathrm{~ms}$ window with the greatest

142 average EMG amplitude within the 5 second contraction, were then used to normalize peak

143 EMG signals obtained during each MVIC trial (Vera-Garcia et al. 2010).

145 Statistical Analysis

146 Paired samples $t$-tests were performed after checking normality using Shapiro-Wilk test in Stata

14713 (StataCorp LP, College Station, TX). Alpha was set a priori at 0.05 for significance. Effect

148 sizes (ES) were calculated by Cohen's $d$ using the formula $d=\frac{M_{d}}{s_{d}}$, where $M_{d}$ is the mean of

149 differences and $s_{d}$ is the standard deviation of differences (Becker 1988; Morris 2007; Smith \&

150 Beretvas 2009). This method is slightly different than the traditional method of calculating

151 Cohen's $d$, as it calculates the within-subject effect-size rather than group or between-subject

152 effect size. ES were defined as small (0.20-0.49), moderate (0.50-0.79), and large ( $\geq 0.80)$

153 (Cohen 1988). Confidence intervals (95\% CI) for each ES were also calculated.

\section{RESULTS}

156 The normalized peak EMG for the different exercises and gluteus maximus sections can be

157 observed in Table 1. In terms of the upper gluteus maximus comparison, no significant

158 differences were observed in the peak EMG for both exercises $(\mathrm{ES}=0.005 ; 95 \% \mathrm{CI}=-0.599-$

$1590.609 ; \mathrm{t}(12)=0.018 ; p=0.986)$. With regards to the lower gluteus maximus, a small ES was 
160 observed $(0.412 ; 95 \% \mathrm{CI}=-0.193-0.102)$ between the two positions in favor of the PRONE

161 position; however, this outcome may have been due to chance alone $(\mathrm{t}(12)=-1.485 ; p=0.164)$.

162

163 Table 1. Group mean \pm SD of normalized peak EMG amplitudes.

\begin{tabular}{ccc} 
& PRONE & SQUEEZE \\
\hline Upper gluteus maximus & $91.94 \pm 11.64$ & $92.04 \pm 11.30$ \\
Lower gluteus maximus & $94.52 \pm 13.59$ & $85.12 \pm 12.64$ \\
\hline
\end{tabular}

164

165

166

\section{DISCUSSION}

167 The purpose of this investigation was to compare a novel gluteus maximus MVIC position,

168 SQUEEZE, to the current gold standard, PRONE. We have failed to reject the null hypotheses,

169 as there were no statistically significant differences between the two positions tested (Table 1).

170 However, despite no statistically significant differences, the peak EMG values for the lower

171 gluteus maximus were approximately $9 \%$ higher for the PRONE compared to the SQUEEZE.

172 Consequently, if the SQUEEZE test were used for normalization, it would render approximately

$17310 \%$ higher mean and peak EMG values compared to the PRONE test. Therefore, although not

174 statistically significant, the findings could be considered practically meaningful. Furthermore,

175 these data show a large amount of individual variation (Table 2), which has been previously

176 described by McGill (1990) and Vera-Garcia et al. (2010) for other muscles. 
178 Table 2. Number of subjects (percentage of subjects (\%)) which each MVIC technique resulted 179 in the greatest peak EMG amplitude.

\begin{tabular}{ccc}
\hline & PRONE & SQUEEZE \\
\hline Upper gluteus maximus & $7(53.85)$ & $6(46.15)$ \\
Lower gluteus maximus & $10(76.92)$ & $3(23.08)$ \\
\hline
\end{tabular}

180

181

182

183

184

185

186

187

188

189

190

191

192

193

194

195

196

197

198

199

200

There are several kinematic and kinetic differences between PRONE and SQUEEZE, any of which may have affected our results, either individually or in combination. During PRONE, the knee is bent to $90^{\circ}$, whereas during SQUEEZE, the knees are fully extended. Previous research has shown that gluteus maximus EMG activity during hip extension is greater with the knees flexed than when extended, presumably resulting from a greater reliance upon the gluteus maximus for hip extension due to decreased hamstrings length (Kwon \& Lee 2013). On the other hand, extended knees allow for greater hip extension range of motion compared to flexed knees, thereby shortening the gluteal fibers to a greater extent (Van Dillen et al. 2000) and leading to a greater amount of gluteus maximus EMG activity (Worrell et al. 2001). In addition, PRONE involved primarily hip hyperextension since the pelvis was fixed, whereas SQUEEZE appeared to involve a combination of hip extension and posterior pelvic tilt. Although posterior pelvic tilt mimics hip extension (Neumann 2010), it is unclear how each of these kinematic variables might affect gluteus maximus EMG activity individually. To our knowledge, no study to date has investigated gluteus maximus EMG activity with varying combinations of hip extension and posterior pelvic tilt during MVIC actions. Moreover, PRONE is an open kinetic chain maneuver with the torso stabilized onto a bench, whereas SQUEEZE is a closed kinetic chain maneuver performed in a standing position. Stensdotter et al. (2003) investigated the EMG activity of the quadriceps muscle group during open kinetic chain and closed kinetic chain positions during MVIC actions and reported significant differences in EMG amplitude. The rectus femoris 
201 displayed greater EMG activity during open kinetic chain maneuvers while the vastus medialis

202 displayed greater EMG activity during closed kinetic chain maneuvers. It is therefore hard to

203 predict whether the gluteus maximus would inherently display greater or lesser EMG activity

204 during either open or closed kinetic chain maneuvers. Finally, PRONE required manual

205 resistance, whereas SQUEEZE relied upon anatomical structures surrounding the hip to provide

206 resistance against hip extension. Whether this factor has any effect on EMG activity recorded in

207 a muscle is unclear, as the authors are unaware of any previous investigations into the effect of

208 squeezing a muscle whereby range of motion is limited by anatomical structures on EMG

209 activity rather than against external resistance.

210

211 This investigation was subject to several important limitations. Firstly, although we observed

212 what may have been a practically important difference between the MVIC positions, this

213 difference was not found to be statistically significant, which suggests that our initial estimates

214 for the appropriate sample size may have been too small. Secondly, there were several kinematic

215 differences between the two positions that were explored (PRONE and SQUEEZE), including

216 different pelvic, hip, and knee joint angles. There were also kinetic differences between the two

217 positions, in that PRONE was an open kinetic chain maneuver and SQUEEZE was a closed

218 kinetic chain maneuver. Moreover, PRONE used external resistance and SQUEEZE utilized

219 oppositional torques produced by internal, anatomical structures. These multiple differences

220 make it difficult to assess whether our results arose from a combination of biomechanical factors

221 acting in opposing directions, heterogeneity, or genuinely no difference between the conditions.

222 Thirdly, we only compared two MVIC positions, and it is feasible that other positions might

223 result in superior or inferior levels of EMG activity. Fourthly, we only investigated two 
224 subdivisions of the gluteus maximus muscle and there are indications that there may be others, 225 from proximal-to-distal, medial-to-lateral, and superficial-to deep. Furthermore, our statistical

226 analysis was not designed to assess whether there was a difference between the EMG activity of

227 the upper and lower gluteus maximus during either MVIC position and therefore this remains 228 uncertain.

\section{Conclusions}

230 Although these data are inconclusive as to which position is superior, they do provide insight as

231 to the complexity of MVIC positions for the gluteus maximus. More specifically, due to the large

232 individual variations (Table 2), it is recommended that multiple MVIC positions be utilized to

233 ensure that the greatest possible EMG amplitude be the divisor during normalization. These

234 recommendations are well in line with other studies, which have utilized or recommended

235 multiple MVIC positions (McGill et al. 2009; Vera-Garcia et al. 2010). Future research should

236 use heterogeneous samples, such as athletic males, and also test more positions, such as the

237 Biering-Sorenson position, quadruped hip extension position, and top hip thrust position

238 (Contreras et al. 2011), each with manual resistance, along with the tall kneeling position. 
241

242

243

244

245

246

247

248

249

250

251

252

253

254

255

256

257

258

259

260

261

262

263

264

265

266

267

268

269

270

271

272

273

274

275

276

277

278

279

280

281

282

Barton CJ, Kennedy A, Twycross-Lewis R, Woledge R, Malliaras P, and Morrissey D. 2014. Gluteal muscle activation during the isometric phase of squatting exercises with and without a Swiss ball. Physical Therapy in Sport 15:39-46.

Becker BJ. 1988. Synthesizing standardized mean - change measures. British Journal of Mathematical and Statistical Psychology 41:257-278.

Boudreau SN, Dwyer MK, Mattacola CG, Lattermann C, Uhl TL, and McKeon JM. 2009. Hipmuscle activation during the lunge, single-leg squat, and step-up-and-over exercises. Journal of Sport Rehabilitation 18:91-103.

Cambridge ED, Sidorkewicz N, Ikeda DM, and McGill SM. 2012. Progressive hip rehabilitation: the effects of resistance band placement on gluteal activation during two common exercises. Clinical Biomechanics (Bristol, Avon) 27:719-724.

Choi SA, Cynn HS, Yi CH, Kwon OY, Yoon TL, Choi WJ, and Lee JH. 2014. Isometric hip abduction using a Thera-Band alters gluteus maximus muscle activity and the anterior pelvic tilt angle during bridging exercise. Journal of Electromyography and Kinesiology.

Cohen J. 1988. Statistical power analysis for the behavioral sciences: Routledge Academic.

Contreras B, Cronin J, and Schoenfeld B. 2011. Barbell hip thrust. Strength \& Conditioning Journal 33:58-61.

Emami M, Arab AM, and Ghamkhar L. 2014. The activity pattern of the lumbo-pelvic muscles during prone hip extension in athletes with and without hamstring strain injury. International Journal of Sports Physical Therapy 9:312-319.

Fischer FJ, and Houtz SJ. 1968. Evaluation of the function of the gluteus maximus muscle. An electromyographic study. American Journal of Physical Medicine 47:182-191.

Fujisawa H, Suzuki H, Yamaguchi E, Yoshiki H, Wada Y, and Watanabe A. 2014. Hip Muscle Activity during Isometric Contraction of Hip Abduction. Journal of Physical Therapy Science 26:187-190.

Hermens HJ, Freriks B, Merletti R, Stegeman D, Blok J, Rau G, Disselhorst-Klug C, and Hägg G. 1999. European recommendations for surface electromyography. Roessingh Research and Development, Enschede.

Hislop H, Avers D, and Brown M. 2013. Daniels and Worthingham's Muscle Testing: Techniques of Manual Examination and Performance Testing: Elsevier Health Sciences.

Jakobsen MD, Sundstrup E, Andersen CH, Aagaard P, and Andersen LL. 2013. Muscle activity during leg strengthening exercise using free weights and elastic resistance: effects of ballistic vs controlled contractions. Human movement science 32:65-78.

Kang SY, Jeon HS, Kwon O, Cynn HS, and Choi B. 2013. Activation of the gluteus maximus and hamstring muscles during prone hip extension with knee flexion in three hip abduction positions. Manual Therapy 18:303-307.

Karlsson E, and Jonsson B. 1965. Function of the Gluteus Maximus Muscle. An Electromyographic Study. Acta Morphologica Neerlando-Scandinavica 6:161-169.

Kendall FP, McCreary EK, Provance PG, Rodgers MM, and Romani W. 1993. Muscles, testing and function: with posture and pain. 
283

284

285

286

287

288

289

290

291

292

293

294

295

296

297

298

299

300

301

302

303

304

305

306

307

308

309

310

311

312

313

314

315

316

317

318

319

320

321

322

323

324

325

326

Kwon YJ, and Lee HO. 2013. How different knee flexion angles influence the hip extensor in the prone position. Journal of Physical Therapy Science 25:1295-1297.

Lyons K, Perry J, Gronley JK, Barnes L, and Antonelli D. 1983. Timing and relative intensity of hip extensor and abductor muscle action during level and stair ambulation. An EMG study. Physical Therapy 63:1597-1605.

McAndrew D, Gorelick M, and Brown J. 2006. Muscles within muscles: a mechanomyographic analysis of muscle segment contractile properties within human gluteus maximus. Journal of Musculoskeletal Research 10:23-35.

McGill SM. 1990. Electromyographic activity of the abdominal and low back musculature during the generation of isometric and dynamic axial trunk torque: implications for lumbar mechanics. Journal of Orthopaedic Research 9:91-103.

McGill SM, McDermott A, and Fenwick CM. 2009. Comparison of different strongman events: trunk muscle activation and lumbar spine motion, load, and stiffness. Journal of strength and conditioning research / National Strength \& Conditioning Association 23:1148-1161.

Morris SB. 2007. Estimating effect sizes from the pretest-posttest-control group designs. Organizational Research Methods.

Neumann DA. 2010. Kinesiology of the hip: a focus on muscular actions. Journal of Orthopaedic and Sports Physical Therapy 40:82-94.

Oh JS, Cynn HS, Won JH, Kwon OY, and Yi CH. 2007. Effects of performing an abdominal drawing-in maneuver during prone hip extension exercises on hip and back extensor muscle activity and amount of anterior pelvic tilt. Journal of Orthopaedic and Sports Physical Therapy 37:320-324.

Simenz CJ, Garceau LR, Lutsch BN, Suchomel TJ, and Ebben WP. 2012. Electromyographical analysis of lower extremity muscle activation during variations of the loaded stepup exercise. Journal of Strength and Conditioning Research 26:3398-3405.

Smith LJW, and Beretvas SN. 2009. Estimation of the Standardized Mean Difference for Repeated Measures Designs. Journal of Modern Applied Statistical Methods 8:27.

Stensdotter AK, Hodges PW, Mellor R, Sundelin G, and Hager-Ross C. 2003. Quadriceps activation in closed and in open kinetic chain exercise. Medicine and Science in Sports and Exercise 35:2043-2047.

Van Dillen LR, McDonnell MK, Fleming DA, and Sahrmann SA. 2000. Effect of knee and hip position on hip extension range of motion in individuals with and without low back pain. Journal of Orthopaedic and Sports Physical Therapy 30:307-316.

Vera-Garcia FJ, Moreside JM, and McGill SM. 2010. MVC techniques to normalize trunk muscle EMG in healthy women. Journal of Electromyography and Kinesiology 20:1016.

Wheatley MD, and Jahnke WD. 1951. Electromyographic study of the superficial thigh and hip muscles in normal individuals. Archives of Physical Medicine and Rehabilitation 32:508-515.

Worrell TW, Karst G, Adamczyk D, Moore R, Stanley C, Steimel B, and Steimel S. 2001. Influence of joint position on electromyographic and torque generation during maximal voluntary isometric contractions of the hamstrings and gluteus maximus muscles. Journal of Orthopaedic and Sports Physical Therapy 31:730-740. 
327 Youdas JW, Foley BM, Kruger BL, Mangus JM, Tortorelli AM, Madson TJ, and Hollman JH. 328 2013. Electromyographic analysis of trunk and hip muscles during resisted lateral 329 band walking. Physiotherapy Theory and Practice 29:113-123. 